\title{
Décadrages Décadrages
}

cınéma, a travers champs Cinéma, à travers champs

$1-2 \mid 2003$

Le hors-champ

\section{Fonctions du regard et du miroir dans Loft Story}

Lecture d'un dispositif de télé-réalité à la lumière de Michel Foucault et Jacques Lacan

Mireille Berton

\section{(2) OpenEdition}

\section{Journals}

Édition électronique

URL : http://journals.openedition.org/decadrages/584

DOI : $10.4000 /$ decadrages. 584

ISSN : 2297-5977

Éditeur

Association Décadrages

Édition imprimée

Date de publication : 1 octobre 2003

Pagination : 35-57

ISSN : 2235-7823

\section{Référence électronique}

Mireille Berton, « Fonctions du regard et du miroir dans Loft Story », Décadrages [En ligne], 1-2 | 2003,

mis en ligne le 26 avril 2013, consulté le 20 avril 2019. URL : http://journals.openedition.org/

decadrages/584 ; DOI : 10.4000/decadrages.584 


\title{
Fonctions du regard et du miroir dans Loft Story \\ Lecture d'un dispositif de télé-réalité à la lumière
}

\section{de Michel Foucault et Jacques Lacan}

\author{
par Mireille Berton
}

"Onze célibataires coupés du monde. Philippe, Laure, Aziz, Loana, Jean-Edouard, Julie, Steevy, Kimy, Fabrice, Kenza, Christophe. Filmés dans un loft de $225 \mathrm{~m}^{2}, 24$ heures sur 24, par 26 caméras et 50 micros. Dans 10 semaines, ils ne seront plus que deux. Qui sera le couple idéal ? C'est vous qui décidez." Tel est le commentaire en voix off qui accompagnait le générique de l'émission Loft Story diffusée sur M6, par satellite sur TPS et sur Internet dès le 26 avril 2001. Cette version édulcorée de «Big Brother», émission de télé-réalité lancée en 1999 aux Pays-Bas, allait alimenter pendant plusieurs mois de nombreux débats sur un phénomène d'une ampleur médiatique sans précédent.

Tout, autour de Loft Story, prendra une tournure hyperbolique, à commencer par les chiffres : 38000 candidats postulants, 100 techniciens mobilisés nuit et jour, des dizaines de caméras et de micros intégrés à un puissant dispositif d'enregistrement, 6 à 10 millions de téléspectateurs, des millions d'appels ou de SMS envoyés chaque jeudi pour sauver son candidat préféré et autant de millions (entre 4 et 12 millions de francs français par semaine) gagnés par M6 et la société de production Endemol.

Au vertige des chiffres répond la fièvre des mots: des quotidiens (Le Monde, Le Figaro, Libération, Le Parisien, etc.), des hebdomadaires à grand tirage (Le Nouvel Observateur, Marianne, L'Express, Télérama, etc.), des revues (Les Cabiers du cinéma, Les Inrockuptibles, Les Dossiers de l'audiovisuel, etc.), des émissions de télévision (Arrêt sur images, Vie privée-Vie publique) ou de radio invitent des journalistes, des sociologues, des historiens de la télévision, des psychanalystes, des philosophes ou autres intellectuels à débattre sur cette curiosité «médiatico-sociologique»1. L'intensité des termes employés reflète suffisamment la force de l'onde de choc provoquée par une émission pourtant bien inoffensive en

1 Ignacio Ramonet, "Big Brother", in Manière de voir, "L'Empire des médias", n 63, mai-juin 2002, p. 30-33. 
2 Sigmund Freud, "Pulsions et destins des pulsions", Métapsychologie (1915), Gallimard (Folio-Essais), Paris, 1997 [1968], p. 11-43.

3 Jean Baudrillard, Télémorphose, Sens \& Tonka, Paris, 2001, p. 25. II ajoute encore à ce propos que "tous les commentaires font eux-mêmes partie du marché culturel et idéologique " qui produit ce genre d'émission d'une insignifiance vertigineuse à son goût. regard de ses avatars ultérieurs: télé-poubelle, fascisme rampant, miracle démocratique, voyeurisme triomphant, jeu concentrationnaire, communion télé-médiatique, esclavagisme moderne, "télémorphose» intégrale de la société, etc., telles sont les expressions qui reviennent assez régulièrement sous la plume des commentateurs.

Deux thèmes principaux se distinguent au sein de ces discours croisant polémiques, discussions et analyses : d'une part, la question de l'énorme succès rencontré par Loft Story, soulevant à son tour une interrogation plus large sur le voyeurisme grandissant d'une société gagnée par le virus de la télé-réalité; d'autre part, le concept oxymorique de "fiction réelle» étudié notamment à l'aide d'instruments importés de théories littéraires ou cinématographiques. L'intérêt se concentre, par conséquent, soit sur la dimension psycho-sociologique du programme, avec comme paradigme principal une théorie du reflet stipulant que notre société a la télévision qu'elle mérite, soit sur la dimension narrative, avec comme objectif la mise à jour d'éventuelles stratégies mystificatrices à l'œuvre dans un «feuilleton-réel» qui va tenir en haleine les téléspectateurs et les internautes durant de longues semaines.

Pour expliquer ce formidable engouement, les analystes, qu'ils soient contempteurs, admirateurs ou froids observateurs, font appel presque invariablement aux mêmes arguments. Reprenant les fameux couples d'opposés décrits dans la Métapsychologie freudienne 2 - voyeurisme/ exhibitionnisme et sadisme/masochisme -, ils mettent sur le compte de nos pulsions les plus élémentaires ce goût immodéré pour la vision d'autrui dans l'exercice de sa quotidienneté, ainsi que celui d'exposer au vu et su de tous son flamboyant anonymat. Ces emprunts lexicologiques hâtifs et souvent approximatifs au champ de la psychanalyse (auxquels nous pouvons encore ajouter les notions de schizophrénie, de paranoïa, de narcissisme, etc.) constituent une première modalité du recours à la "science de l'inconscient", la seconde étant repérable dans les discours traitant de la pertinence fonctionnelle et éthique du rôle des psychologues et psychiatres engagés dans l'opération à titre de garde-fous. Dans les deux cas, c'est une psychanalyse réduite à sa plus simple et simpliste expression qui est sommée d'apporter des réponses sur les dérives psychologiques d'une société diagnostiquée comme malade, intoxiquée par des images dégradantes portant atteinte à la dignité humaine.

Prenant le risque de participer à cette "contagion mystérieuse, cette chaîne virale qui fonctionne d'un bout à l'autre, et dont nous sommes complices jusque dans l'analyse» $\mathbf{3}$, nous nous proposons d'apporter ici un éclairage quelque peu différent sur un objet télévisuel «inaugural» qui présente l'avantage, sur toutes les formes successives de télé-réalité fondées sur les principes de l'enfermement et/ou de l'interactivité (Nice 
People, Kho-Lanta, Star Academy, etc.), de déployer un dispositif suffisamment intéressant, c'est-à-dire transparent et naïf, pour esquisser une réflexion plus générale sur les rapports complexes que l'individu entretient à la fois avec son image et celle d'autrui.

\section{Pour une étude du dispositif loftien}

Il s'agira donc pour nous de pratiquer un chemin très partiellement balisé jusqu'ici, celui d'une étude du dispositif télévisuel mis en place par les concepteurs de Loft Story, d'un dispositif qui peut être appréhendé à la fois comme une machinerie physique et matérielle (un plateau de télévision géant doté d'éclairages, de caméras, de micros, de miroirs en nombre inhabituel; une régie adjacente au plateau centralisant le fonctionnement de ces appareils d'enregistrement et de vision; des engins et des lieux de réception en connexion avec ce dispositif), et comme une machinerie métapsychologique fondée sur une économie particulière des flux psychiques et perceptifs. Plus précisément nous tenterons de comprendre, dans une perspective psychanalytique, quelles sont les implications d'un tel dispositif dans la construction d'une subjectivité nécessairement médiatisée par l'image, le regard et la voix d'autrui situés en lieu et place de l'Autre.

Par conséquent nous nous pencherons tout particulièrement sur le hors-champ de ce dispositif, le cinquième dans la typologie burchienne des espaces hors-champs au cinéma, celui qui se situe "derrière la caméra » $\mathbf{4}$, c'est-à-dire celui qui correspond au lieu de fabrication des images, espace habituellement invisible au spectateur. En ce qui concerne le dispositif de Loft Story, le hors-champ se décline sous plusieurs formes, le hors-champ principal - le lieu de confection des images - ayant plusieurs ramifications.

En effet, c'est essentiellement une régie centrale qui filme les images, enregistre le son, module l'éclairage, dirige les activités des lofteurs par messages écrits et voix off interposés, élabore des résumés quotidiens diffusés sur M6, oriente la lecture de ces images par le biais du montage et d'intertitres, conçoit l'émission hebdomadaire en prime time, règle la diffusion du programme en continu et «en direct» (avec $2 \mathrm{~min} .45 \mathrm{sec}$. de retard autorisant la pratique d'une censure) sur le bouquet numérique TPS et sur Internet. Bref, ce hors-champ tentaculaire qui exerce le contrôle éditorial de ces différents produits 5 a comme point d'ancrage une régie où s'affairent des dizaines de techniciens et de réalisateurs chargés d'élaborer les différents épisodes de ce feuilleton d'un nouveau type.

Pour simplifier, nous pouvons diviser ce hors-champ en deux instances principales corrélatives l'une de l'autre: le hors-champ de la production qui se présente aux candidats du jeu comme étant «Le
4 Noël Burch, "Nana ou les deux espaces", Une praxis du cinéma, Gallimard (Folio/Essais, $n^{\circ}$ 34), Paris, 1986 [1969], p. 30.

5 François Jost, "Un nom, trois produits", L'empire du loft, La Dispute, Paris, 2002, p. 52-55. 
6 L'Autre, notion repérable à toutes les étapes de la réflexion lacanienne, présente un degré de polysémie qui rend malaisée toute tentative de définition univoque. Comme le souligne P.-L. Assoun, elle est employée "pour désigner des figures diverses, voire hétérogènes, en sorte qu'il est légitime de se demander: qu'est-ce que l'Autre?". Paul-Laurent Assoun, Lacan, PUF (QSJ?, n 3360), Paris, 2003, p. 63. Nous reviendrons sur cette question au cours de notre exposé.

7 Pour être très succinct: I'Imaginaire, sans rapport direct avec l'imagination, désigne le rapport à l'image du semblable et au corps propre, tout en constituant une conception originale du narcissisme défini comme une forme particulière d'investissement libidinal du Moi; le Sym bolique, distinct du symbolisme, renvoie au langage, aux règles de la vie en société, à la Loi, et donc également à l'inconscient conçu comme un savoir ayant la structure d'un langage composé d'une chaîne de signifiants; le Réel, qui n'est pas du tout superposable à la réalité, représente tout ce qui demeure en dehors du Symbolique, tout ce qui ne peut être saisi par le langage: il est le lieu de l'impensable, de l'informulable, de l'inconnaissable, de l'impossible. Lire à ce propos la conférence donnée au Congrès de Rome, "Fonction et champ de la parole et du langage en psychanalyse" (1953), in Jacques Lacan, Ecrits I, Seuil, Paris, 1970 [1966], p. 111-208.
Propriétaire", instance abstraite, désincarnée mais néanmoins omniprésente, et le hors-champ de la diffusion qui correspond au public qui suit les péripéties de Loft Story sous une forme ou sous une autre, tout en étant intégré au dispositif par le biais des votes destinés à éliminer les candidats au fil des semaines. Ainsi, le champ (la vie à l'intérieur du Loft), le hors-champ de la production et celui de la consommation de ce programme fonctionnent, sur un plan à la fois narratif, psychologique et économique, en étroite interdépendance.

L'analyse de ce dispositif et de son hors-champ s'opérera en deux temps principaux. Dans une première phase, nous tenterons de procéder à une analyse du fonctionnement de ce dispositif, notamment, à partir de l'examen d'une séquence paradigmatique qui condense tous les éléments permettant de dégager une problématique relative à la primauté du regard. Nous mettrons alors en évidence un réseau de relations inter- subjectives qui se nouent autour de la vision chez les occupants respectifs du champ et du hors-champ, trajets qui sont soutenus, de part en part, par des fantasmes liés à la pulsion scopique, à un désir de voir, cependant sans rapport direct avec le voyeurisme. Nous montrerons alors que ce sont ces modalités d'échanges de regards et de désirs entre les différents champs qui, en définitive, assurent la fonctionnalité d'un dispositif et celle d'un jeu fondé sur la centralité du regard. De plus, il s'agira de tracer la filiation historique de ces désirs dépendants de la fonction visuelle en remontant à une "source" première probable, celle du panoptisme (et du panoramisme). Situer le Loft dans une histoire de la vision et de la représentation au plus long terme nous permettra d'ébaucher ainsi une définition socio-historique d'un spectacle en apparence inédit, puisque la télé-réalité ne fait, en définitive, que mettre les nouvelles technologies au service d'idéologies plus anciennes.

Afin de mesurer plus exactement la portée d'une telle circularité de désirs, de demandes et de regards entre ces différentes sphères d'influence que sont le champ et ses hors-champs, nous articulerons, dans un second temps, la conception de hors-champ, telle qu'elle a été définie plus haut, au concept de l'Autre, notion élaborée par Jacques Lacan et désignant ce dont dépend le sujet, c'est-à-dire le lieu de ses déterminations (le langage), ce à quoi il fait recours et ce qui est invoqué pour mettre de l'ordre dans le monde humain ${ }^{6}$. En effet, un des pôles principaux de la pensée lacanienne consiste en une dramaturgie organisée autour des trois dimensions de la théorie du désir: celle de l'Autre, de l'objet et du sujet. Ces notions à leur tour ne peuvent être comprises sans leur imbrication dans les trois registres de la réalité humaine désignés comme étant l'Imaginaire, le Symbolique et le Réel7. Nous mettrons en 
évidence le rôle joué par l'image, l'imaginaire et le spéculaire dans la construction du Moi et de la subjectivité. Il s'agira par conséquent d'inscrire cette analyse dans le cadre de la théorie de l'Imaginaire inaugurée en 1936 par le fameux texte de Lacan sur le stade du miroir ${ }^{\mathbf{8}}$, théorie qui ne cessera de subir des reformulations successives pour aboutir à une théorie structurale du spéculaire.

Ce rapprochement théorique que nous opérons entre le hors-champ du dispositif télévisuel à l'œuvre dans Loft Story et la fonction de l'altérité dans la construction d'un sujet aliéné au champ de l'Autre, se fonde sur une analogie résidant dans le référent spéculaire. En effet, si Loft Story fait des miroirs et des glaces sans tain un emploi massif, Lacan, dans le sillon tracé par Freud qui décrivait l'appareil psychique comme une structure plurisystémique semblable à un instrument de vision ${ }^{9}$, fait, lui aussi, régulièrement appel à des métaphores optiques - et en particulier celle du miroir - pour rendre compte de la topique subjective. Nous verrons comment, dans les deux cas, les miroirs fonctionnent comme pivot entre deux lieux fonctionnellement "inégaux» au regard de la "vérité" du sujet: un champ, lieu de l'imaginaire, et un hors-champ, lieu du symbolique, qui soumet le champ à ses impératifs. Nous invitons donc le lecteur à lire le registre de l'Autre comme une sorte de "hors-champ" de l'individu, antérieur à son avènement en tant que sujet, et absolument déterminant dans l'élaboration des idéaux de la personne.

\section{Le dispositif loftien et le motif de l'œil}

Revenons donc au point de départ, celui que nous nous sommes fixé avec la prise en considération du dispositif loftien, en examinant de plus près une séquence emblématique de l'émission, à savoir celle du générique. En effet, cette séquence, qui revient invariablement en début de chaque diffusion télévisuelle, présente un double intérêt. En premier lieu, elle donne en quelques secondes, aussi bien au niveau de l'image que du verbal, un résumé des conditions du jeu et de ses règles $\mathbf{1 0}$. En quelques mots et images, tout est dit: le générique permet de répondre à la fois au quoi, qui, où, pendant combien de temps, pourquoi et pour qui d'un tel programme, les mots clé de l'énoncé venant s'inscrire sur l'écran, au côté des images, en lettres blanches sur un fond noir.

Cet exposé synthétique du principe de l'émission s'apparente à la trame narrative minimale d'un scénario qui prescrit un cadre aux candidats, tous des acteurs "non professionnels» invités à jouer leur propre rôle. Les données matérielles du dispositif, la temporalité, le but du jeu et l'interactivité imposent au "réel» des contraintes qui forment ainsi une sorte d'écriture scénaristique dictée par la production.

Les images du générique, quant à elles, participent à cette même $\mathbf{8}$ Ce texte, censé être présenté par Lacan lors du Congrès psychanalytique international de Marienbad (1936), a été perdu, mais sera repris par l'auteur en 1949, à l'occasion du $X V \mid l^{e}$ Congrès international de psychanalyse à Zurich, sous le titre "Le stade du miroir comme formateur de la fonction du ije, telle qu'elle nous est révélée par l'expérience psychanalytique", conférence qui sera publiée plus tard dans les Ecrits (1966). Cf. Jacques Lacan, Ecrits I, op. cit., p. 89-97.

9 En septembre 1899, Freud aborde la partie la plus théorique de L'Interprétation des rêves avec le célèbre chapitre VII consacré à la métapsychologie du rêve. S'efforçant de définir le psychisme comme l'espace constitutif du rêve, il emploie alors une métaphore à la fois optique et photographique, en comparant l'appareil psychique au microscope, au télescope et à l'appareil photographique. Sigmund Freud, L'interprétation des rêves (1900), PUF, Paris, 1971, p. 455.

10 Cf. infra. 


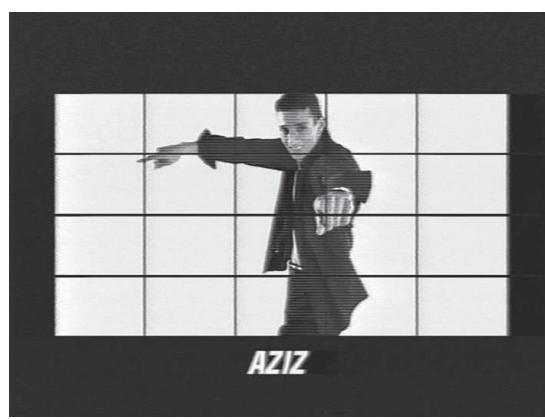

1

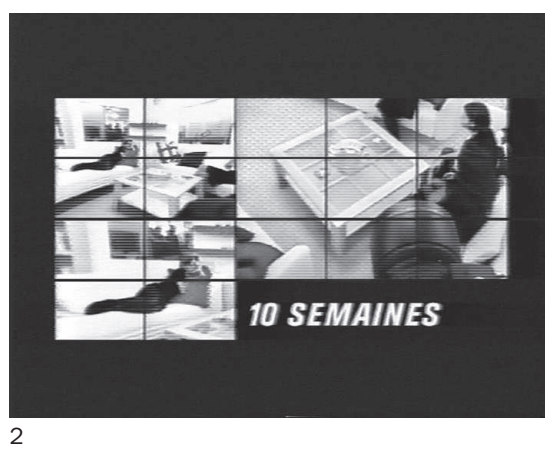

11 Christian Metz, Essais sur la signification au cinéma, t. 2, Klincksieck, Paris, 1972.

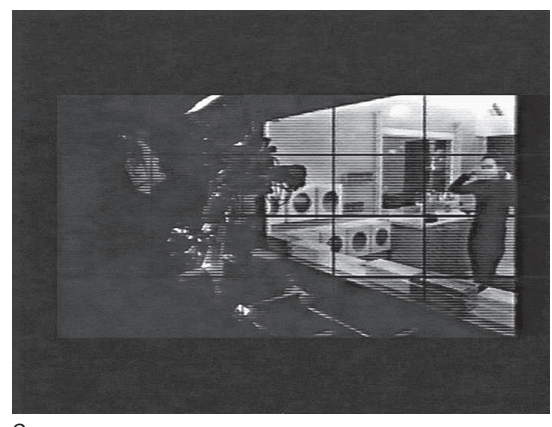

3 logique condensatoire et fictionalisante puisqu'elles montrent successivement et en synchronie avec le texte dit en voix off une série de scènes diverses à l'intérieur du Loft habité par des locataires fictifs, ainsi qu'une vue aérienne du Loft, des caméras, des micros, des plans sur la régie et ses techniciens, séquence qui se conclut avec des images d'un couple (hypothétique) formé à la fin de l'aventure. Cette séquence répond, à certains égards, à la définition donnée par Christian Metz de la «séquence par épisodes" reposant sur un enchaînement chronologique de scènes emblématiques séparées par de fortes sautes spatio-temporelles. Chaque image apparaît dès lors comme un "résumé symbolique d'un stade dans une évolution assez longue que la séquence globale condense» $\mathbf{1 1}$.

Par ailleurs, si cette séquence repose sur le principe d'un montage très court entre les plans, elle se structure également sur un montage interne à l'image dont les modalités changent lorsque les cases dont est composé l'écran varient (fig. 1). En effet, l'écran de télévision coïncide partiellement avec un cadre finement divisé en vingt rectangles par des droites noires, horizontales et verticales qui découpent l'écran en plusieurs zones, elles-mêmes divisées en différentes images, qu'elles soient semblables ou différentes (fig. 2). Or, cette conception graphique d'une surface quadrillée d'images rappelle la configuration d'un dispositif "multi-écrans", couramment utilisé dans les espaces de vidéo-surveillance. En reproduisant symboliquement un dispositif de surveillance, ce choix formel indique assez clairement l'orientation d'une émission fondée sur les ressources d'une vision totalisante et d'un fantasme d'omniperception. Un des plans sur la régie où figure une série d'écrans vidéo superposés vient d'ailleurs explicitement souligner cette allégeance au principe panoptique qui consiste à embrasser d'un seul coup d'œil la vaste étendue d'un lieu.

Tel est le second point qui mérite d'être soulevé à propos de ce générique. En effet, cette séquence introductive constitue l'unique occasion pour le téléspectateur de voir des images concrètes du dispositif qui se déploie, non pas devant, mais derrière les caméras, c'est-à-dire d'avoir un aperçu, même bref, sur l'espace dévolu à la confection des images. Celleci s'effectue, au stade du filmage, grâce au maniement de trois types de caméras : dix-sept caméras fixes (dont des caméras infrarouges dans les chambres à coucher), neuf caméras télécommandées et quatre caméras (maniées manuellement) se déplaçant sur des rails encerclant le Loft sur 160 mètres de long. Une image du générique nous montre précisément un technicien manipulant une caméra sur rail, dissimulée derrière une glace sans tain, afin d'obtenir des images du Loft dont on perçoit partiellement l'intérieur à travers cette "fenêtre" translucide (fig. 3). Ce plan nous montre donc simultanément le «dedans» et le «dehors» d'un 
dispositif séparé par une série de «membranes» à fonction réversible. En effet, une observation attentive des lieux démontre que pratiquement toutes les parois, et a fortiori toutes les pièces (y compris l'enceinte du jardin), sont munies, "côté face", de miroirs à première vue destinés à nourrir le narcissisme exacerbé de candidats exhibitionnistes, et "côté pile", d'écrans semi-transparents qui permettent de voir et de filmer sans être vu.

Cette séquence initiale présente d'autres éléments de nature autoréflexive consolidant la promesse de tout voir et de tout entendre proférée par les producteurs: les images d'objectifs de caméra zoomant (fig. 4); les points de vue plongeants avec, en amorce, une caméra de surveillance (fig. 2); les mains de techniciens réglant l'intensité du son en régie; les images floues, tramées, pixelisées d'écrans vidéo, etc. Tous ces plans font office de monstration ostentatoire, d'exhibition d'un

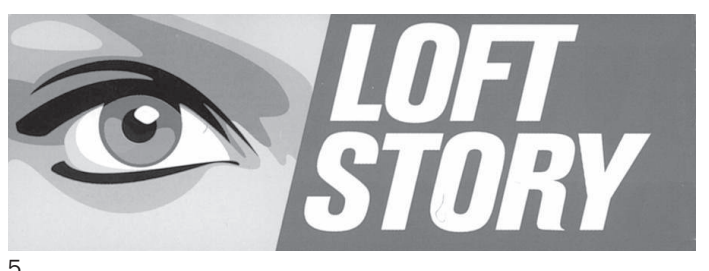

dispositif technologique d'enregistrement relayé par une multitude d'appareils perceptifs d'une efficacité redoutable. Une image essentielle, toutefois, incontournable et répétitive, les subsume tous: celle de l'œil "virtuel» coloré qui sert de logo, de marque de fabrique à l'émission, mais qui fonctionne aussi comme un signe de ponctuation entre les séquences, tout en inscrivant de manière insistante, dans l'esprit du téléspectateur, l'idée que ce dernier se situe du côté du voir plus que du percevoir (fig. 5). On est en droit, en effet, de se demander si une oreille n'aurait pas mieux fait l'affaire étant donné l'extrême pauvreté "télégénique» d'une vie de réclusion et d'oisiveté, comparativement à l'«attractivité» d'une logorrhée patente et permanente menée par des lofteurs désœuvrés. Mais l'entêtant renvoi à l'optique poursuit le téléspectateur jusque dans le clignement de l'œil qui s'accompagne à chaque fois d'un bruit d'obturateur - ce mariage entre l'organe de la vue et la photographie représentant certainement une référence inconsciente aux avant-gardes artistiques des années 20 qui aimaient à penser l'appareil photographique comme un prolongement visuel technique permettant d'étendre et d'accroître les possibilités de l'œil humain.

Le regard, on le constate, occupe une place tout à fait prépondérante au sein d'un dispositif qui nourrit et entretient deux sortes de fantasmes fondés sur la pulsion scopique prise dans un sens très général, sans 
12 Un des malentendus majeurs concernant la psychanalyse freudienne (et donc aussi lacanienne) réside dans la réduction de la sexualité à une activité génitale, alors que le sexuel freudien concerne, pour simplifier, la vie "pulsionnelle" de manière générale. La sexualité ne désigne donc pas uniquement le plaisir dépendant des organes génitaux, mais tout un ensemble d'activités présentes dès l'enfance et qui procurent une satisfaction irréductible au simple assouvissement d'un besoin physiologique. Le champ du sexuel mérite donc d'être étendu à une définition moins "biologique" et plus large de la sexualité, étant entendu que tout être humain est "traversé par l'aspiration constante et jamais réalisée, à atteindre un but impossible, celui du bonheur absolu, bonheur qui revêt différentes figures dont celle d'un hypothétique plaisir sexuel absolu ". J.-D. Nasio, Cinq leçons sur la théorie de Jacques Lacan, Payot-Rivages, Paris, 1994 [1992], p. 35.

13 Parmi ces auteurs citons Jean Baudrillard (Télémorphose, op. cit.), Ignacio Ramonet ("Big Brother ", in Manière de voir, op. cit.) et Vincent Charbonnier, chargé de cours et doctorant à I'Université de Nantes (article sur Internet: http://loftscary.free.fr/ca06.htm).

14 François Jost, "Qui a peur de Big Brother?", L'empire du loft, op. cit., p. 13-32.

15 Bernard Comment, Le XIXe siècle des panoramas, Adam Biro, Paris, 1993, p. 9.

16 Michel Foucault, Surveiller et punir. Naissance de la prison, Gallimard, Paris, 1975. aucune implication érotique ${ }^{\mathbf{1 2}}$ : du côté de l'instance productrice et consommatrice, on peut repérer, non pas tant un fantasme voyeuriste ni même sadique, mais un désir d'omniperception qui vise à atteindre une maîtrise inégalée de la fonction perceptive par des moyens technologiques modernes et sophistiqués. L'objet de ce fantasme d'omnivoyance, c'est-à-dire le groupe des candidats captifs de ce dispositif de type panoptique, en plus d'être gagné par une très forte compulsion d'enfermement, est animé, quant à lui, par un fantasme de complétude identitaire (et imaginaire) étroitement dépendante du regard de l'autre.

Nous allons donc évaluer successivement les modalités et les implications de tels fantasmes.

\section{Le fantasme d'omniperception : panoptisme et panoramisme}

La remarque concernant l'homologie entre le dispositif du Loft et celui du modèle carcéral, le Panopticon (fig. 6), conçu par Jeremy Bentham en 1791 comme un lieu dans lequel chaque détenu est enfermé dans une cellule individuelle d'où il est observé par un surveillant placé dans une tour centrale, autorisant ainsi le déploiement d'un regard circulaire et englobant, n'est certes pas totalement inédite ${ }^{\mathbf{1 3}}$. Cependant, un seul auteur, François Jost, a pris la peine d'analyser les réelles implications d'un tel rapprochement pour aboutir à la conclusion que le Loft (et d'autres dispositifs similaires tels les webcams) se présente comme une sorte de panoptique inversé dans la mesure où il ne s'agit plus de mettre en jeu un seul regard couvrant un champ occupé par une multitude d'individus, mais de conférer le pouvoir à une masse d'exercer une vision inquisitrice sur un petit nombre de personnes exposées au regard de tous $\mathbf{1 4}$. Si cette lecture nous paraît tout à fait pertinente, nous aimerions néanmoins revenir en détail sur les enjeux d'une telle parenté, et tenter de situer le Loft dans la continuité historique "d'un désir particulièrement vif au XIX ${ }^{\mathrm{e}}$ siècle, celui d'une maîtrise absolue qui procure à chaque individu le sentiment euphorique que le monde s'organise autour de lui et à partir de lui, un monde dont il est en même temps séparé et protégé par la distance du regard»15.

Depuis l'examen du principe du panoptisme effectué par Michel Foucault dans sa généalogie des prisons, les modalités de fonctionnement de ce dispositif disciplinaire et de ses dérivés sont bien connues $\mathbf{1 6}$. Née à la fin du XVIII ${ }^{\mathrm{e}}$ siècle, cette maison pénitentiaire, dont le modèle perdurera jusqu'à nos jours, met en scène une "boucle» de détenus au centre de laquelle un gardien s'emploie à observer le pourtour sans être vu lui-même (fig. 7). Ce système présente l'avantage d'induire chez le prisonnier "un état conscient et permanent de visibilité qui assure le fonctionnement automatique du pouvoir", et ceci en faisant en sorte 


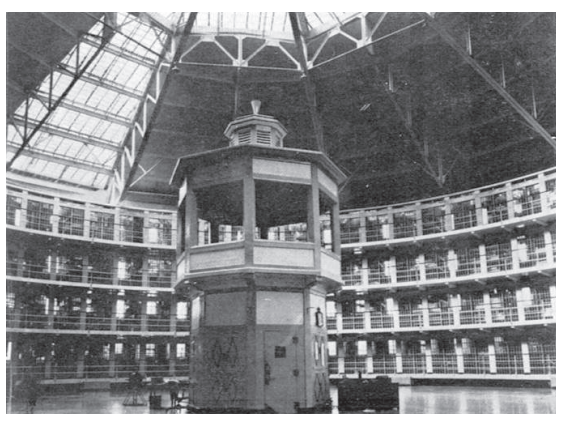

JEREMY BENTHAM, PLAN ET COUPE POUR LE PANOPTICON (1791)

INTÉRIEUR DU PÉNITENCIER DE STATEVILLE, ETATS-UNIS, $X X^{e}$ SIÈCLE

que «la surveillance soit permanente dans ses effets, même si elle est discontinue dans son action „17. L'efficacité du système panoptique dépend donc de deux conditions essentielles: d'une part, le détenu doit avoir continuellement conscience du dispositif d'où il est épié, et d'autre part, il ne doit jamais vraiment savoir à quel moment précis il est regardé. Le Panoptique représente donc une réelle «machine à dissocier le couple voir-être vu: dans l'anneau périphérique, on est totalement vu, sans jamais voir, dans la tour centrale, on voit tout sans être vu»18. Cette dramaturgie des regards implique donc qu'il y ait une maîtrise inégale de la vision et de la visibilité, une dissymétrie entre le regardé et le regardant, et ceci d'autant plus que la fonction de surveillant peut être assumée par n'importe qui, pourvu que le détenu garde toujours à l'esprit qu'il est susceptible d'être observé à tout instant. En effet, "la curiosité d'un indiscret, la malice d'un enfant, l'appétit de savoir d'un philosophe qui veut parcourir le museum de la nature humaine, ou la méchanceté de ceux qui prennent plaisir à épier et à punir», tous ces types de regards sont autorisés à s'exercer dans le cadre de cette "machine merveilleuse qui, à partir des désirs les plus différents, fabrique des effets homogènes de pouvoir»19.

Ce modèle d'enfermement qui gère de manière simple et précise la «distribution concertée des corps, des surfaces, des lumières et des regards »20, qui active une économie du flux visuel à la fois déséquilibrée et discriminante, qui permet l'expérience d'une pulsion scopique aux motivations les plus diverses et qui automatise, désingularise et inocule le pouvoir de sorte que "celui qui est soumis à un champ de visibilité [...] reprend à son compte les contraintes du pouvoir» sans les remettre en question, ce système présente des similitudes frappantes avec le dispositif loftien. En effet, le lofteur intègre très vite les règles implicites dictées par un espace rempli de caméras, de micros, de miroirs, baigné d'une lumière artificielle puissante qui l'oblige à porter des lunettes de soleil à l'intérieur comme à l'extérieur, le poussant ainsi à se transformer

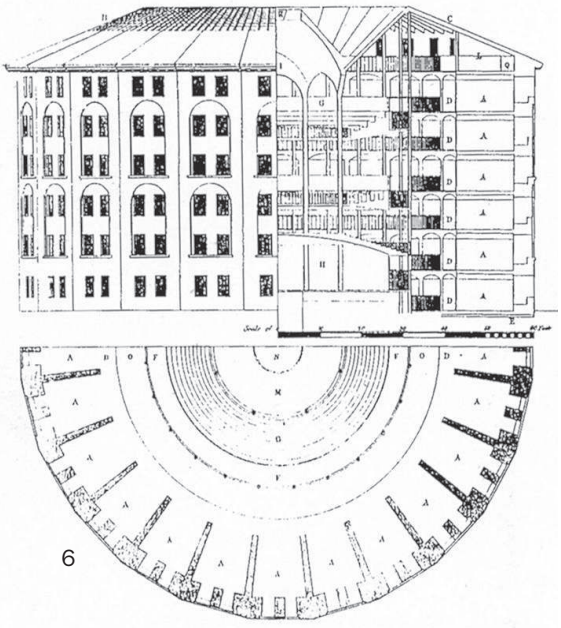

17 /d., p. 202.

18 ld., p. 203.

$19 / d .$, p. 204.

20 ld., p. 203. 
$21 / d .$, p. 204.

22 ld., p. 205-206.

23 ld., p. 209.

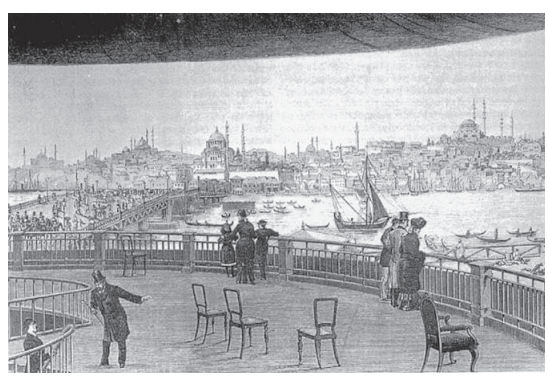

8

PLATE-FORME ET DÉTAIL D'Un PANORAMA (CELUI DE CONSTANTINOPLE, PAR J. GARNIER) EXPOSÉ À AMSTERDAM DANS LES ANNÉES 1880 lui-même en spectacle permanent, car là aussi, comme dans le Panoptique, «un assujettissement réel naît mécaniquement d'une relation fictive. De sorte qu'il n'est pas nécessaire d'avoir recours à des moyens de force pour contraindre le condamné à une bonne conduite» $\mathbf{2 1}$.

$\mathrm{Si}$, dans les prisons, il est recommandé d'opter pour une ligne de conduite moralement irréprochable, les impératifs du Loft, d'un autre ordre, invitent les candidats à adopter une humeur enjouée, toujours à la fête, jamais morose pour amuser un public télévisuel né dans la société $\mathrm{du}$ spectacle où règne la dictature du sourire et de l'esprit gouailleur. Le Loft, comme le Panopticon dans ses variantes scolaires ou hospitalières, constitue aussi un laboratoire destiné à mener des expériences sur un groupe social visant à "modifier le comportement, à dresser ou redresser les individus [...], à analyser en toute certitude les transformations qu'on peut obtenir sur eux»22. Qu'il s'agisse d'expérimentations sur des criminels, des malades, des enfants ou sur la jeunesse française contemporaine, l'enjeu est toujours le même: servir à la fois d'exemplum pour le peuple, d'un peuple qui, dans le cas du Loft, cherche à s'identifier à un semblable dont le mérite est d'avoir pu prendre sa revanche sur l'écrasante et sordide inertie quotidienne, ainsi que de laboratoire pour l'agent du pouvoir, le "Propriétaire», qui use de ce schéma carcéral pour mieux asseoir son influence paternaliste et assurer la pérennité économique de l'entreprise. Comme le note Foucault, si ce modèle de coercition subtile va se diffuser à la société entière dès le XVIII ${ }^{\mathrm{e}}$ siècle, c'est parce qu'il répond à un besoin, celui de «rendre plus fortes les forces sociales» ${ }^{23}$, d'augmenter la production, de développer l'économie et d'édifier la masse.

De manière plus générale, l'idée d'un regard circulaire, englobant et omnipercevant qui sous-tend une véritable vogue des architectures circulaires au XVIII ${ }^{e}$ et au XIX ${ }^{e}$ siècle, va s'étendre à d'autres phénomènes pour donner naissance au panoramisme et à son corollaire, le centrement du sujet embrassant dans un regard continu et totalisant un monde qui s'offre à lui. Comme le panorama (fig. 8) - cette peinture circulaire apposée sur la paroi intérieure d'une rotonde et qui, contemplée par un spectateur placé sur une plate-forme centrale, donne une très forte impression de réalité - le dispositif loftien assigne lui aussi au spectateur une place fixe et intransgressable, offre, par le biais d'un simulacre de réalité, un spectacle qui se substitue à l'expérience vécue, nourrit le fantasme d'une perception globale et instille le sentiment euphorique d'omniscience. Dans les deux cas, "l'individu se place [...] en position de maîtrise par rapport à l'espace environnant et sur le double axe du temps et de la distance». Mais, tout comme le statut du sujet percevant du panorama, celui du téléspectateur est paradoxal car «la maîtrise à 
laquelle il accède, suppose son propre effacement et la perte du lieu réel pour adhérer au lieu fictif de la représentation». Le Loft façonne lui aussi un public dispensable qui doit se contenter d'accéder à une représentation biaisée de la réalité puisqu'elle est découpée, censurée, reconstituée par le montage à l'œuvre dans des résumés quotidiens qui ne retiennent que les instants prégnants - tout comme les panoramistes sélectionnaient les épisodes les plus significatifs au moment de peindre le déroulement d'une bataille. Dans le panorama, comme dans le Loft, «cette ellipse est la condition de l'illusion et de sa pleine efficacité» $\mathbf{2 4}$.

\section{Un dispositif de surveillance ludique}

Si le dispositif du Loft s'écarte singulièrement de la disposition en cercle et du principe du regard continu présents dans le panoramisme pour adopter un éclatement "arachnéen" des points de vue disséminés dans un espace adoptant le plan «traditionnel» de tout appartement moderne (fig. 9), il n'en demeure pas moins qu'il en reprend l'idéologie fondamentale. Répétons-le, l'émission ne fait que reproduire «un dispositif typique de contrôle (policier, carcéral, militaire), renforcé par l'élimination des angles morts, la multiplication des plongées, les caméras infrarouge $[. .$.$] , qui donne au spectateur une sensation de puissance, de$ maîtrise, de domination, et en même temps renforce à la longue le sentiment protecteur à l'égard des enfermés volontaires »25. D'un point de vue historique donc, le Loft semble constituer le point de convergence

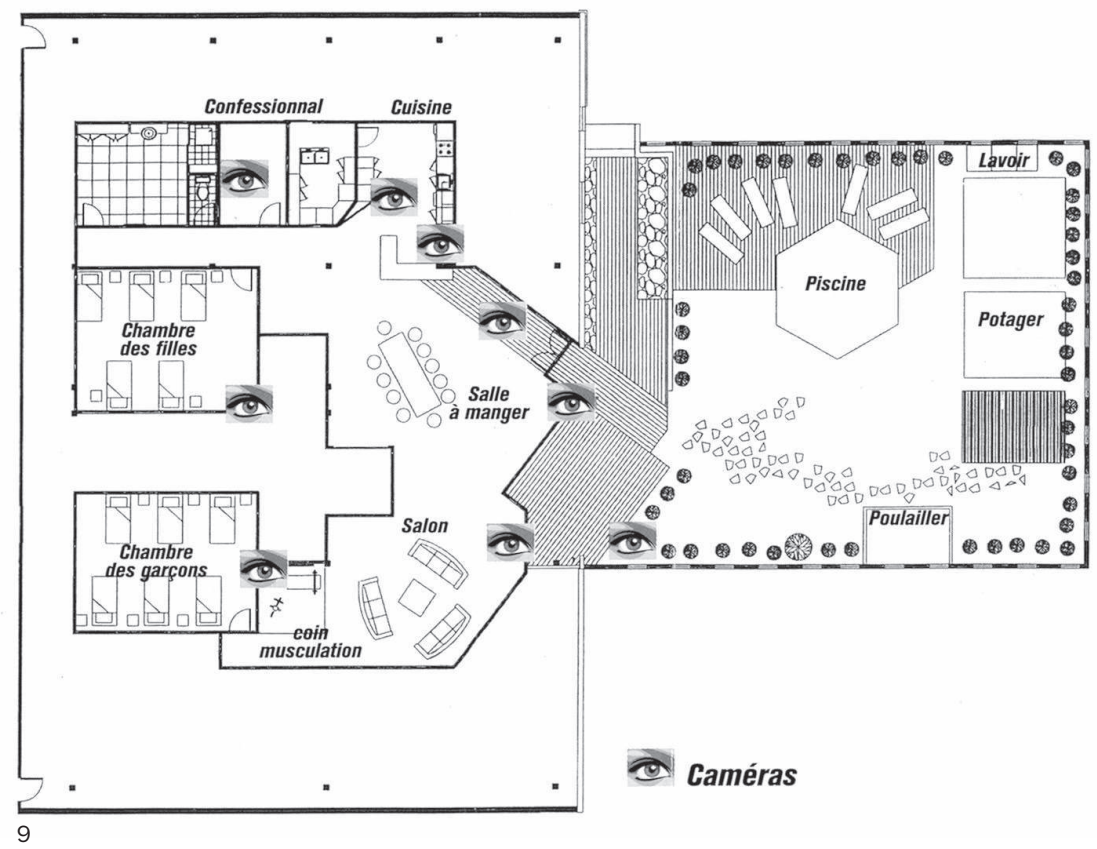

24 Bernard Comment, Le XIXe siècle des panoramas, op. cit., p. 96-97. Dans le Loft, cette abolition des limites spatio-temporelles trouve son expression la plus parfaite dans la revendication d'une transmission en direct et dans la promesse, maintes fois répétée par l'animateur Benjamin Castaldi, d'une restitution complète, " $24 \mathrm{~h}$ sur $24 \mathrm{~h}$ ", "jour et nuit", de tous les événements du Loft. Pour une critique des promesses non tenues par la production lire François Jost, "La réalité télévisuelle", L'empire du loft, op. cit., p. 33-66.

25 Ignacio Ramonet, "Big Brother", in Manière de voir, op. cit. 
26 Roger Caillois, Les jeux et les hommes. Le masque et le vertige, Gallimard, Paris, 1967 [1958].

27 Pour une histoire de la télé-réalité et des genres télévisuels, lire François Jost, La télévision du quotidien. Entre réalité et fiction, De Boeck Université, Bruxelles, 2001.

28 Michel Foucault, Surveiller et punir, op. cit., p. 218.

29 Roger Caillois, Les jeux et les hommes, op. cit. entre deux époques ou traditions: d'une part la tradition des spectacles antiques fondée, entre autres, sur le goût du simulacre et de la compétition $\mathbf{2 6}$, et d'autre part la tradition moderne de «spectacles» de surveillance dirigés par un Etat interventionniste appliquant une logique sécuritaire. En effet, si Michel Foucault repère une transition historique avec le passage, au tournant du XIX ${ }^{\mathrm{e}}$ siècle, d'une forme de spectacle héritée de l'Antiquité - où la vision d'un nombre réduit de choses est rendue possible à une multitude de spectateurs - vers une configuration spectaculaire moderne qui vise à procurer à une minorité de personnes la vue simultanée d'un nombre élevé d'objets, il nous faut toutefois remarquer que cette tentation du spectacle de masse, consommé autour d'une minorité d'individus chargés de distraire cet immense et informe corps spectatoriel, semble connaître un regain de faveur avec l'avènement de la télé-réalité27.

$\mathrm{Au}$ spectacle antique, le Loft emprunte le rapport quantitativement inégal entre public et acteurs, l'interactivité, "l'intensité des fêtes, la proximité sensuelle» à l'œuvre dans des "rituels où coulait le sang", permettant ainsi à la société de retrouver vigueur en formant «un grand corps unique» $\mathbf{2 8}$. Au flot de sang a fait place un flot de larmes systématiquement déclenché à l'annonce solennelle de l'expulsion du candidat pourtant "nominé» un peu plus tôt par ceux-là mêmes qui le pleurent et le regrettent déjà, et ceci tout en favorisant chez le public le processus d'une catharsis collective. De plus, les jeux d'imitation, de simulation, de relookage, de travestisme au sein du Loft sont légion et rappellent l'omniprésence de la mimicry (simulacre) propre aux sociétés primitives où règnent le masque et la pantomime ${ }^{29}$. Néanmoins, Loft Story présente une forme fortement dégénérée de la mimicry primitive puisque, avec le passage à la civilisation moderne, les fonctions de métamorphose et les valeurs religieuses rattachées au masque ont été progressivement neutralisées et apprivoisées pour les vider de tout leur sens.

$\mathrm{Au}$ spectacle panoptique de l'ère moderne, le Loft reprend l'idée d'une rationalisation de l'espace favorisant la domestication d'individus qui se plient de bonne grâce à une logique concentrationnaire visant, entre autres choses, une mutation morale et physique des êtres incarcérés.

Le Loft réalise donc une sorte de synthèse idéologique entre les spectacles antiques et le panoramisme moderne avec l'appui des technologies de communication modernes qui autorisent, non seulement le déploiement d'un regard englobant, mais aussi la diffusion d'un programme "plus-que-parfait», puisque ses concepteurs garantissent au public, non pas simplement une illusion de réalité (comme c'etait le cas avec le panorama du XIX ${ }^{e}$ siècle), mais une illusion de vérité procurée par des candidats qui ne jouent qu'à être eux-mêmes. Ainsi, la télé-réalité apparaît 
comme un symptôme d'un phénomène plus ample sous-tendu par une recherche accrue de vérité, au détriment d'une réalité dont les ressources semblent avoir été épuisées par l'histoire de la représentation. Depuis quelques décennies, en effet, l'ensemble des programmes télévisés (et pas uniquement les unités de divertissement) privilégient le témoignage à l'information traditionnelle, l'interview au compte-rendu journalistique ${ }^{30}$, car l'authenticité d'un témoin qui a vu et vécu mérite plus de crédit qu'une analyse à froid et objective ${ }^{\mathbf{3 1}}$. Avec le Loft, nous assistons donc à la réconciliation entre la société du spectacle telle qu'elle a été définie par Guy Debord, et la société de surveillance décrite par Foucault, cette hybridation coïncidant avec l'émergence d'une société qui aime à mélanger la pratique du jeu aux angoisses sécuritaires. Plus que d'une télévision misant sur l'amalgame entre fiction et réalité, le Loft découle d'une stratégie visant à produire un spectacle de surveillance ludique qui renverse totalement les principes de la logique disciplinaire, puisque les prisonniers se pressent pour se livrer à leurs bourreaux et craignent à tout moment d'être libérés de leurs chaînes $\mathbf{3 2}$.

\section{Au-delà du miroir: le regard de l'Autre}

Afin de cerner plus précisément la situation particulière de ces détenus volontaires, il nous faut à présent mesurer les retombées métapsychologiques d'un tel dispositif en explorant la configuration des rapports qui s'instituent entre les habitants du Loft et un environnement artificiel anormalement lumineux, réflexif, scruté et scrutateur, mais également entre un hors-champ symbolique et un champ imaginaire médiatisé par des caméras et des miroirs intervenant comme des opérateurs d'identité. Il s'agira alors d'évaluer la portée de l'influence que peut exercer un tel dispositif sur des individus traqués aussi bien par le regard de l'autre (celui des objectifs de caméras), que par le regard propre (celui du reflet spéculaire).

Au sein du Loft, une des activités préférées des locataires consiste à se regarder dans le miroir, soit pour des raisons fonctionnelles (se laver, se raser, se (dé)maquiller, se percer un bouton, se coiffer, etc.) (fig. 10), soit pour des motifs plus simplement «narcissiques» comme la vérification de l'adéquation de sa tenue, de la mise en place d'une perruque, de l'effet bluffant d'un déguisement ou du style ravageur d'une chorégraphie. Parmi toutes ces occupations, ils semblent en affectionner une tout particulièrement, puisqu'ils consacrent beaucoup de temps à danser ou à bouger individuellement ou en groupe devant les miroirs, et si possible devant ceux qui restituent la Gestalt en son entier, comme dans la salle à manger. L'injonction permanente à faire la fête pour divertir la plèbe n'est qu'en partie responsable de ce rituel étrange qui peut se produire
30 D'ailleurs, aujourd'hui le présentateur du télé-journal congédie systématiquement un envoyé spécial ayant présenté son reportage par un "merci pour ce témoignage". Ainsi, les journalistes eux-mêmes sont réduits à n'être plus que des simples témoins rendant compte d'une réalité médiatisée par une subjectivité paradoxalement garante de vérité, à l'égal des invités de C'est mon choix, un talk show qui confère le droit à la parole "vraie" au simple quidam.

31 Lire à ce sujet, François Jost, La télévision du quotidien, op. cit.

32 Dans ce sens F. Jost a raison de souligner qu'aujourd'hui plus personne n'a peur de Big Brother, l'instance persécutive et omnisciente, décrite par George Orwell dans 1984, préfiguratrice des modèles d'observation à distance; désormais les dispositifs de surveillance remplissent une fonction inverse puisqu'ils sont destinés à rassurer le citoyen, à le protéger contre les agressions du monde extérieur. Dans cette perspective, le Loft peut être comparé à l'environnement intra-utérin qui, à la fois, met l'individu à l'abri des chocs trop directs en provenance de la réalité, et le berce d'un sentiment océanique (Kristeva) qui préserve l'illusion d'une complétude subjective.

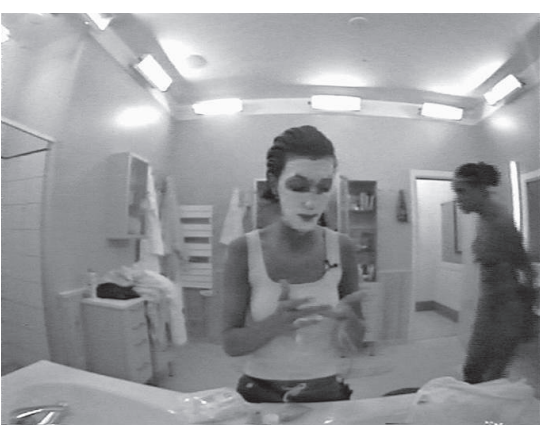


33 Jean Baudrillard, Télémorphose, op. cit., p. 92-93. inopinément, au détour des moments les plus creux de la vie communautaire. Les candidats semblent, en tous les cas, ressentir un besoin régulier d'affronter leur propre regard, de se jauger, de s'assurer de leur être-au-monde par le biais d'une image.

L'omniprésence des miroirs paraît ainsi inviter tout naturellement le lofteur à se regarder très souvent et sans retenue aucune dans un espace quadrillé de surfaces réflexives. Il lui est donc difficile d'échapper à son image, de se détourner volontairement d'un reflet "persécuteur» qui, de plus, a la faculté de se démultiplier dans un espace totalement ceinturé de miroirs (il est possible, par exemple, de se voir simultanément de face et de dos). Cette cohabitation forcée et prolongée avec l'image de soi ne peut, certes, être supportée sans incident majeur que par des individus nourrissant à l'égard de leur propre personne des sentiments pleins d'aménité. En optant pour une interprétation plus sombre de cette intimité spéculaire, nous pouvons affirmer, avec Baudrillard, qu'«il ne s'agit plus tellement de sauvegarder un territoire symbolique que de s'enfermer avec sa propre image, de vivre en promiscuité avec elle comme dans une niche, en complicité incestueuse avec elle, avec tous les effets de transparence et de retour-image qui sont ceux d'un écran total, et n'ayant plus avec les autres que des rapports d'image à image» $\mathbf{3 3}$.

Toutefois, ce goût prononcé pour l'auto-inspection ne doit pas, à notre sens, être mis uniquement sur le compte d'un narcissisme surdéveloppé chez des personnes décomplexées. Ces regards sur soi qui viennent s'inscrire sur l'«endroit» de la surface spéculaire sont en fait redoublés sur leur «envers" par des regards «invisibles» en provenance du hors-champ, c'est-à-dire par les regards de ceux qui les observent grâce aux caméras situées derrière les glaces sans tain, regards auxquels s'ajoutent, par déplacement et sous une forme médiatisée, ceux des techniciens en régie qui visionnent ces images et ceux du public devant son poste de télévision qui a alors l'impression de se situer derrière la «vitre» semi-transparente séparant les deux espaces in et off.

Dans le cadre du Loft, cette pratique fréquente du face-à-face avec l'image spéculaire n'exprime pas seulement un simple penchant pour l'auto-contemplation, mais également une volonté inconsciente d'obtenir un «retour» sous la forme d'une consolidation identitaire. En effet, cette «émission» de flux scopiques dépend du lieu fonctionnant comme "caisse de résonance", d'un lieu qui, matériellement, correspond à l'audelà des miroirs, c'est-à-dire au hors-champ du dispositif, mais qui, sur un plan psychique, coïncide avec une instance abstraite reconduisible au champ de l'Autre. Selon nous, la théorie de Lacan sur l'Imaginaire et l'avènement du sujet peut fournir un éclairage inédit sur cette dialectique de regards (ou de non-regards), permettant ainsi de dépasser 
"l'impasse» du narcissisme, notion à laquelle les commentateurs font régulièrement appel pour justifier la récurrence de ces comportements exhibitionnistes.

\section{L'aliénation du moi au regard de l'Autre: de l'Imaginaire au Symbolique}

La perception de soi dans le miroir, acte en apparence anodin, présente en effet des implications beaucoup plus complexes qu'il n'y paraît. Le Loft, avec son dispositif saturé de miroirs, vient signifier l'importance du stade du miroir comme témoignant de la puissance de fascination de l'image, mais aussi de la force de captation par l'image et de ses effets leurrants, effets qui perdurent la vie durant ${ }^{\mathbf{3 4}}$. Le stade du miroir, en rappelant la dette que tout individu contracte auprès d'un tiers qui vient authentifier la découverte de soi, permet de comprendre le rôle décisif joué par l'Autre dans la construction de son identité. Entre le lofteur et son image dans le miroir - image à la fois formatrice et aliénante - se glisse un troisième terme qui, bien qu'invisible, médiatise le rapport que l'individu entretient avec lui-même et avec autrui. Symbolisant le lieu qui brise l'illusion psychologique du sujet, l'Autre a pour fonction de signifier que "le sujet n'est pas sa propre origine", qu'avant qu'il «ne ressente quelque chose ou n'entre en relation avec un objet, l'Autre est Ce qui est déjà là. On comprend aussi que la 〈rencontre〉 de l'Autre se fera dans les occurrences diverses où le sujet s'éprouve déstabilisé en son ipséité»35. Cet Autre prend, dans notre analyse du dispositif loftien, la figure d'un Autre située au-delà des miroirs et des objectifs de caméras, dans un hors-champ symbolique qui englobe les "autres imaginaires" que sont le public et la production ${ }^{\mathbf{3 6}}$. L'image que renvoie le miroir n'est qu'un reflet imparfait, incomplet, dépendant du regard, du désir et du savoir de l'Autre qui seul a le pouvoir de lui donner sa véritable consistance. "Parce qu'il est une <image` projetée à travers ses multiples représentants, le Moi ne peut prendre sa valeur de représentation imaginaire que par l'autre et au regard de l'autre »37. Ainsi, l'identification du lofteur à son image spéculaire n'est rendue possible que dans la mesure où elle est soutenue d'une certaine reconnaissance dans l'Autre.

Cet Autre est à référer au registre du Symbolique. Structurel et rhétorique, l'ordre symbolique ou linguistique est ce qui préexiste au sujet individuel et continue d'exister après sa disparition. L'ordre symbolique est doublement indépendant du sujet individuel: d'une part, parce qu'il n'existe que collectivement, et d'autre part parce qu'il est pour une très large part inconscient dans le sujet individuel même. C'est en ce double sens que le symbolique est l'Autre qui conditionne l'avènement du sujet individuel. Par conséquent, Lacan affirme que le Symbolique constitue précisément le discours de l'Autre, cet Autre qui est le lieu de la parole,
34 La phase du miroir est habituellement conçue comme constituant le moment fondateur de I'Imaginaire, un des trois ordres à l'œuvre dans la vie psychique du sujet, et qui comprend le répertoire d'images que le sujet invoque pour annuler le fossé originaire entre la réalité et le fantasme. Cet ordre est présent dans la phase du miroir en tant qu'image de l'autre à laquelle l'enfant s'identifie, tout en permettant de masquer la division de l'être. La phase du miroir réalise une première ébauche du moi qui se constitue sur la base de l'identification à l'image du semblable sous la forme d'une unité corporelle, identification primordiale qui constituera la matrice de toutes les identifications ultérieures (les identifications secondaires) par lesquelles se structurera la personnalité du sujet. Jacques Lacan, "Le stade du miroir comme formateur de la fonction du ije, telle qu'elle nous est révélée par l'expérience psychanalytique", Ecrits I, op. cit., p. 89-97.

35 Paul-Laurent Assoun, Lacan, op. cit., p. 64.

36 Lacan prend soin de distinguer l'autre (avec une minuscule) qui appartient au registre de I'Imaginaire, et l'Autre (avec majuscule) qui relève du Symbolique. Cf. D'un Autre à l'autre, Séminaire de 1968-1969.

37 Joël Dor, Introduction à la lecture de Lacan, Denoël, Paris, 2002 [1985], p. 156. 
38 Philippe Julien, Pour lire Jacques Lacan, Seuil, Paris, 1990 (1985), p. 68-69.

39 Paul-Laurent Assoun, Lacan, op. cit., p. 64. qui accueille tout ce qui se prononce et s'est prononcé. En venant au monde, l'enfant s'inscrit dans un univers symbolique qui détermine sa place. "Or, à cet ordre se subordonne l'ordre imaginaire; la parole de nomination en l'Autre [...] vient se conjoindre à la vision de l'autre. L'aliénation imaginaire par laquelle le sujet voit son désir sur l'image de l'autre, se double de l'aliénation symbolique par laquelle le désir du sujet est reconnu comme désir de l'Autre» $\mathbf{3 8}$.

En effet, l'identification implique l'aliénation au désir de l'autre, car elle est sanctionnée par un autre, depuis un autre lieu que lui-même. L'énoncé «c'est moi» est vérifié non pas par l'enfant, mais par un adulte, habituellement la mère (l'Autre), qui vient confirmer de l'extérieur cette impression d'identité. L'enfant s'identifie donc avec la perception qu'a sa mère de lui-même ou plutôt à ce qu'il imagine que la mère désire qu'il soit. En disant «c'est moi», l'enfant dit «je suis un autre». Ainsi, le désir est toujours destiné à passer à travers le désir de l'Autre. En effet, si c'est l'autre qui lui dérobe son désir, l'autre ne peut que refléter un désir qui devient alors le désir de l'autre. Cela implique donc une aliénation et une division du sujet qui doit accepter une identité qui a été déterminée ailleurs, par l'ordre symbolique du langage. Devenant et demeurant "désir du désir de l'autre», désir du désir de sa mère, désir de combler le manque de sa mère, l'individu, bien qu'acquérant le langage, n'accède jamais vraiment à l'ordre symbolique. Ce que désire l'homme, c'est que l'autre le désire, c'est devenir ce qui manque à l'autre, c'est être l'origine du désir de l'autre. Mais cette coïncidence entre son désir et le désir de l'autre est impossible à réaliser en raison de la méconnaissance du sujet et des barrières dressées par l'Imaginaire qui font de lui le jouet névrosé des images captivantes de lui-même que les autres lui tendent. "Introduire l'Autre, c'est donc récuser l'autonomie de l'imaginaire pur ou, en d'autres termes, rappeler la détermination de l'imaginaire par le symbolique »39. L'enfant, fondamentalement divisé dès le moment où se forme une ébauche de son Moi, va chercher une compensation au manque à être dans l'unité fictive offerte par le miroir, mais n'y trouvera qu'une division supplémentaire recouvrant et compliquant le manque originel. Il s'établit dès lors un fossé irréductible entre la réalité de l'être forcément incomplet et aliéné, et l'image idéalisée que l'enfant se fait de lui-même.

\section{Les lofteurs en quête de complétude subjective}

Les candidats du Loft, au lieu d'acquérir une nouvelle identité médiatique qui les hisse au statut de vedettes, tout en les confortant dans l'idée d'une complétude identitaire, ne font que traverser, dans des conditions particulièrement spectaculaires, cette expérience originelle 
de l'aliénation au regard, à la parole et au désir de l'Autre que l'enfant, devant le miroir, prend à témoin pour obtenir un assentiment concernant son identification primordiale. En effet, le dispositif du Loft met en évidence avec brio l'inféodation du Moi comme construction imaginaire à la dimension de l'Autre: les multiples regards jetés vers les miroirs peuvent être décodés, sur leur versant inconscient, comme des appels lancés au lieu de l'Autre, comme des "projections" du Moi vers le lieu symbolique situé hors-champ, un lieu à la fois en dehors de soi et connecté au sujet par des solutions imaginaires leurrantes. Qu'il se trouve face à son image propre ou face à un autre que lui, le sujet est toujours pris dans les filets de l'imaginaire (le Moi) qui demandent à être "dénoués» dans l'ordre symbolique (l'Autre). Ainsi, "dans la relation intersubjective, quelque chose de factice s'introduit toujours qui est la projection imaginaire de l'un sur le simple écran que devient l'autre »40.

Que ces écrans de projection soient les miroirs, les locataires du Loft, le «Propriétaire», le public, la mère (les «maman, je t’aime» criés face aux caméras l'attestent), peu importe. Toutes ces figures sont à placer du côté de l'Autre, «cet être supposé» qui peut être "n'importe quel personnage mythique que ce soit Dieu, la mère, ou le sujet lui-même dans un fantasme de toute-puissance» $\mathbf{4 1}$. Lieu utopique pour la psychanalyse et mythique pour le sujet, l'Autre ne reste pas moins la figure à laquelle s'adressent, à son insu, ses regards et ses paroles.

Cette dépendance fondamentale du sujet vis-à-vis de l'Autre est à mettre en rapport avec la labilité, la précarité et l'incomplétude d'une identité acquise au fil des processus identificatoires, et fondée sur le fait que la reconnaissance de soi n'est jamais directe puisqu'elle passe par une autre image qui demeure en son extériorité, même une fois intériorisée. L'image réelle de soi échappe donc inévitablement au sujet qui ne peut se reconnaître que dans une image "virtuelle» renvoyée par le miroir que constitue l'autre. C'est ce que Lacan a tenté d'expliquer avec la métaphore optique du vase renversé, qui reprend la dialectique spéculaire introduite dans le stade du miroir, mais pour la "compliquer» et ajouter au miroir plan un miroir concave $\mathbf{4 2}$. Ce schéma optique permet notamment à Lacan de démontrer que le sujet est un être foncièrement divisé, clivé, qu'il ne se voit jamais de là où il se regarde. En effet, "si d'une certaine manière, c'est dans l'Autre qu'il se voit, c'est également dans l'espace de l'Autre que vient se situer le point d'où il se regarde » $\mathbf{4 3}$.

"En effet, le sujet virtuel, reflet de l'œil mythique, c'est-à-dire l'autre que nous sommes, est là où nous avons d'abord vu notre ego - hors de nous, dans la forme humaine [...]. L'être humain ne voit sa forme réalisée, totale, le mirage de lui-même, que hors de lui-même " $\mathbf{4 4}$. Il y a ainsi une logique d'exclusion entre l'œil et le regard qui rend le sujet captif
40 Roland Chemena, Dictionnaire de la psychanalyse, Larousse, Paris, 1995, p. 140.

41 J.-D. Nasio, Cinq leçons sur la théorie de Jacques Lacan, op. cit., p. 38-39.

42 Le dispositif comporte un miroir concave qui réfléchit sur un miroir plan l'image d'un bouquet renversé sous un vase, de sorte que dans le miroir plan l'observateur voit le bouquet dans le vase. Dans l'impossibilité de décrire de manière extensive cette métaphore, nous renvoyons le lecteur aux multiples références à celle-ci dans Les écrits techniques de Freud. Le Séminaire, Livre I (1953-1954), Seuil, Paris, 1998 (1975), ainsi qu'au chapitre ("Le schéma optique et les idéaux de la personne. Moi idéal et Idéal du moi ") consacré à ce sujet dans Joël Dor, Introduction à la lecture de Lacan, op. cit., p. 304-326.

43 Joël Dor, Introduction à la lecture de Lacan, op. cit., p. 322.

44 Jacques Lacan, "Idéal du moi et Moi idéal", Les quatre concepts fondamentaux de la psychanalyse. Le Séminaire, Livre XI (1964), Seuil, Paris, 1990 [1973], p. 221. 
45 Maurice Merleau-Ponty, La Phénoménologie de la perception, Gallimard, Paris, 1945.

46 Jacques Lacan, "La schize de l'œil et du regard", Les quatre concepts fondamentaux de la psychanalyse. Le Séminaire, Livre XI (1964), op. cit., p. 87

47 Michel Thévoz, Le miroir infidèle, Minuit, Paris, 1996, p. 10. d'une méconnaissance chronique. Reprenant les acquis de la phénoménologie de Merleau-Ponty 45, Lacan affirme la préexistence du regard sur l'œil: le sujet est regardé avant d'être regardant, tout comme l'être est parlé avant d'être parlant. Le sujet percevant doit donc se soumettre à cette primauté d'une omnivoyance qui le regarde, avant qu'il ne puisse luimême regarder. "Nous sommes des êtres regardés dans le spectacle du monde», regardés par un «regard qui nous cerne» et qui fait apparaître le monde comme fondamentalement omnivoyeur. Ainsi, affirme Lacan, «je ne vois que d'un point, mais dans mon existence, je suis regardé de partout» 46 .

Le dispositif du Loft présente ainsi une exemplarité hors du commun, car non seulement il illustre le fantasme d'omnivoyance (et d'omniperception) qui travaille une partie de l'histoire de la représentation, ainsi que la société moderne du "spectacle sécuritaire», mais il offre également une traduction à la théorie psychanalytique du sujet comme "voyant ou visible au sein de l'universelle 〈voyure»», comme un sujet qui se fait «regard qui quête ou provoque le regard de l'autre dans une insoluble alternative» 47 .

Plus généralement, on peut avancer que ce désir d'une poignée d'individus d'être regardés (et donc aimés) par des millions de téléspectateurs constitue une sorte de pléonasme puisqu'il ne fait que "répéter", redoubler maladroitement la condition de tout être au monde, celle d'être un objet de regard avant tout, d'être soumis à l'antériorité du regard de l'Autre. Le dispositif du Loft met en scène de manière ostentatoire le désir qui anime tout sujet, celui d'un désir de reconnaissance, d'un désir qui trouve son sens dans le désir de l'Autre, car précisément ce que le désir vise c'est d'être reconnu par cet Autre.

\section{Les lofteurs en quête de certitude identitaire}

Dans leur quête effrénée de reconnaissance médiatique, les candidats à la notoriété éphémère du jeu Loft Story sont aussi, de manière inconsciente, des candidats à la complétude subjective, état d'achèvement sanctionné par le regard et le désir du public qui, à son tour, projette ses fantasmes de réussite sur un écran de télévision. Pourtant, dans ces échanges intersubjectifs, seules des images biaisées, déformées, inadéquates de soi sont reçues par autrui, car le désir d'être reconnu comme tel est un désir fondamentalement aliéné qui n’appartient pas vraiment en propre à l'individu, mais qui est le désir de sa part divisée avec laquelle il ne peut pas faire totalité.

Durant le jeu, les lofteurs se montrent particulièrement soucieux de renvoyer une image "juste" et vraie d'eux-mêmes à ceux qui les regardent. Le thème du "décalage identitaire", en effet, obsède la 
plupart d'entre eux qui craignent d'être incompris, mal jugés ou méprisés par les autres en raison d'une mauvaise "communication" d'image de soi. Pour preuve, le départ de David qui, au bout de cinq jours, crée un choc dans la petite communauté en révélant qu'il n'a, à aucun moment, été "lui-même», qu'il n'a fait que jouer la comédie, qu'il s'est donc fait passé pour un autre. Il s'est donc rendu coupable de haute trahison par ceux qui s'imaginent n'avoir dupé personne en maintenant des rapports sincères et loyaux, aussi bien vis-à-vis des autres, que visà-vis d'eux-mêmes. Sur un autre plan, le cas Loana, la gagnante du jeu, illustre un autre effet d'aliénation puisque chez elle, c'est un décalage flagrant entre son apparence (une belle fille siliconée et toujours légèrement vêtue) et son essence (une fille sensible, meurtrie par la vie et dotée d'un quotient intellectuel aussi impressionnant que son tour de poitrine), qui va beaucoup intriguer ses congénères. Jean-Edouard, pour sa part, souffre du dédain des filles choquées par son attitude de goujat, après sa «rupture» avec Loana qui, de son côté, a cru voir en lui l'homme de sa vie. Dans le confessionnal, il déclare alors n'être qu'un homme "normal» qui ne refuse pas un moment de plaisir, si on le lui propose si gentiment. Kenza, invitée de Mireille Dumas dans Vie privée, vie publique après sa sortie du Loft, s'offusquera du fait que les résumés de M6 restituent très faiblement l'authenticité des faits et de la psychologie profonde des candidats.

Cette volonté permanente de résorber l'écart entre une image de soi supposée "vraie» et une image flottante, reçue et octroyée par l'autre, corrobore parfaitement le fait que le sujet va toujours chercher dans l'autre l'image réelle de soi-même, mais que cette quête identitaire connaît de multiples contrariétés qu'il s'agit de surmonter par différentes stratégies visant à rectifier, à «mettre au point» l'image floue et infidèle qui a été émise dans un premier temps de manière "involontaire». Tel est le danger engendré par le fonctionnement proprement tribal de cette petite "famille» (c'est ainsi qu'ils se qualifient): dans ce constant déploiement du privé sur la place publique, le phénomène d'une perte d'identité de soi semble inévitable puisqu'aujourd'hui, sur les plateaux de télévision, l'individu est pensé par les autres, n'existe que par l'autre et à condition de se confondre dans l'autre $\mathbf{4 8}$.

\section{La soumission à la parole structurante de l'Autre}

Mais, si le sujet dépend du regard de l'Autre, il dépend également de la parole et de la voix de l'Autre qui s'inscrit, nous l'avons vu, dans le registre du Symbolique. Cette fonction invocante de l'Autre constitue le "pendant auditif» de la fonction scopique: le sujet parlant adresse des appels, des demandes, des questions à l'Autre qu'il perçoit comme
48 Michel Maffesoli, Le temps des tribus: le déclin de l'individualisme dans les sociétés de masse, Méridiens Klinscksieck, Paris, 1988. 
49 J.-D. Nasio, Cinq leçons sur la théorie de Jacques Lacan, op. cit., p. 226.

50 Alain Vanier, Lacan, Les Belles lettres, Paris, 2000, p. 19.

$\mathbf{5 1}$ Jacques Lacan, "Fonction et champ de la parole et du langage en psychanalyse ", Ecrits I, op. cit., p. 181. étant le détenteur de sa "vérité», d'une vérité méconnue, dérobée à laquelle il cherche à accéder par son truchement. En effet, selon Lacan, toute parole appelle une réponse car il n'y a pas de parole sans Autre, l'Autre primordial étant notre langue maternelle, le lieu à partir duquel nous avons pu parler et nous construire. Ainsi, au sein du Loft, cette quête d'identité passe aussi par la parole, par les échanges verbaux, par les révélations en aparté proférées dans le confessionnal vers un lieu situé hors-champ, discussions qui tournent toutes presque toujours autour des mêmes questions: qui est-il vraiment, m'aime-t-elle un peu, tu me trouves comment, que pensent-ils vraiment de ma personne, dis-moi quels sont mes défauts? etc. À chaque fois, ces interrogations demeurent en suspens, en attente au lieu de l'Autre censé contenir quelques réponses. "La demande de savoir, c'est le propre de la névrose. Le névrosé se définit par le fait que sa demande, nette, non ambiguë, est d'un savoir; il veut savoir, il veut que l'Autre lui parle et lui apprenne»49. Sans vouloir dresser un tel profil psycho-pathologique des lofteurs, il s'agit ici de mettre en évidence le rôle vital de la parole au sein d'un dispositif fonctionnant comme un piège à regards. Lacan attribue en effet une fonction tout à fait positive à la parole qui permet de sortir de l'impasse imaginaire, car «le rapport du sujet à son image dans le miroir conduit à une difficulté propre à la dimension narcissique. Cette capture conduit à une situation mortifère du type: ou l'un ou l'autre. Pour que le lien social soit possible, il faut concevoir un autre terme, qui ne laisse pas le sujet dans une relation strictement en miroir à un semblable. Ce qui arrive dans le mythe, à Narcisse se noyant pour rejoindre sa propre image, indique assez la limite du modèle. Cet élément médiateur, cette dimension tierce, qui sort le sujet de l'impasse imaginaire, c'est la parole et le langage»50. Ainsi, si l'Autre est le lieu du regard, il est aussi et surtout le lieu de la parole, le lieu qui reçoit la dialectique sans fin du désir dont le premier but est d'être reconnu par l'autre. "Ce que je cherche, dit Lacan, dans la parole c'est la réponse de l'autre. Ce qui me constitue comme sujet, c'est ma question»51. Le sujet loftien, continuellement submergé d'interrogations relatives à sa personne, démontre de manière tragi-comique la validité de cette maxime lacanienne.

Si la parole a donc une fonction structurante pour le lofteur, elle n'en demeure pas moins le lieu de malentendus, de rencontres manquées, car les aléas de la communication intersubjective proviennent en particulier du fait que la parole a une fonction essentiellement invocatrice et non informative. Dans un Loft coupé (partiellement) du monde, où les journaux, la télévision et la radio sont interdits, où la lecture est restreinte au strict minimum, où le seul débat contradictoire consiste à savoir si les filles seront toutes jetées dans la piscine pour un bain de 
minuit collectif ou alors si Aziz est un macho ou non, les conversations finissent inévitablement par revenir sur les mêmes interrogations, les mêmes doutes et les mêmes thèmes - les difficultés (ou les joies) des relations affectives - et cela sur le mode de la confession intime, de la révélation de soi aux autres, autant d'échanges qui s'avèrent "sans issue", car induits et sciemment exploités par le "Propriétaire», un Autre qui a pris soin de sélectionner des personnes suffisamment malléables psychologiquement pour obtenir le résultat voulu: une obéissance aveugle au désir et à la loi de l'Autre ressenti comme une instance omnisciente et toute-puissante.

Dans le Loft, la parole de l'Autre prend une autre forme que celle des échanges entre les candidats. En effet, le «Propriétaire» du Loft, situé dans un hors-champ à la fois prestigieux et improbable, a coutume de communiquer avec ses locataires par le biais de messages en voix off ou de messages écrits remis dans le cellier, pièce médiatrice entre l'intérieur et l'extérieur. Cette voix et cette parole impersonnelles, changeantes, décorporées 52 assument vis-à-vis des lofteurs le rôle de l'Autre, d'une instance qui façonne leurs comportements, qui impose des activités, qui juge des performances, qui récompense une victoire, qui punit une faute, etc. À leur tour, les candidats s'adressent ou répondent aux questions de l'Autre dans le confessionnal, lieu capitonné, à l'écart des regards et oreilles indiscrètes des autres lofteurs, qui place le pénitent face à une caméra, favorisant ainsi la délation, l'aveu, la doléance, la déclaration, l'accusation, la contrition, etc. Tous ces actes de paroles sont reçus à la fois par le fameux «Propriétaire» et par le téléspectateur qui s'identifie alors au confesseur bénéficiant du privilège d'entendre ces révélations dans un tête-à-tête factice, qui tout à la fois le rend complice et le met à distance, l'écran de télévision ayant remplacé la grille (fig. 11). Ce que le téléspectateur ne perçoit pas, car situé hors-champ et coupé au montage, c'est que ces mots censés relever de la plus libre expression, constituent en fait des réponses à des questions précises qui induisent inévitablement une transformation des propos en un discours orienté par la personne chargée de mener la "confession». Cet escamotage de l'Autre comme instance invocante ne fait que renforcer l'illusion d'une parole subjective autonome.

Cette commun(icat)ion improbable souligne l'aporie d'un langage où «notre message nous vient de l'Autre, et pour l'énoncer jusqu'au bout: sous une forme inversée». Ce principe s'applique à toute forme d'énonciation, "puisqu'à avoir été émis par nous, c'est d'un autre, interlocuteur éminent, qu'il a reçu sa meilleure frappe»53. Tous les mots prononcés dans le Loft par caméras interposées ou non rappellent de manière aiguë cette formule lacanienne: les êtres parlants ne sont jamais totalement $\mathbf{5 2}$ Ce terme est utilisé par les psychanalystes pour désigner un processus de désinvestissement corporel ou d' "évanouissement" de l'enveloppe corporelle.

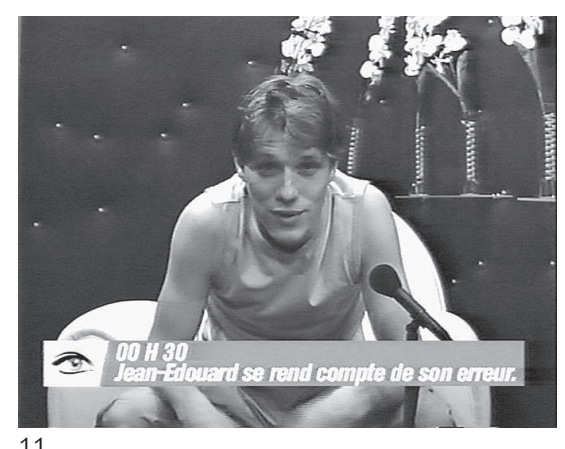

11

53 Jacques Lacan, "Ouverture de ce recueil", Ecrits I, op. cit., p. 15-16. 
54 Joël Dor, Introduction à la lecture de Lacan, op. cit., p. 101.

$\mathbf{5 5}$ Lire à ce sujet le très beau texte de Michel Thévoz, "L'inversion spéculaire", Le miroir infidèle, op. cit., p. 19-35.

$56 / d .$, p. $27-28$.

57 ld., p. 20.

58 /d., p. 30. maîtres de leurs dits, ils émettent des paroles dans «le vide» du champ de l'Autre qui les renvoie inlassablement à leurs destinataires.

\section{Le malentendu originel: l'inversion de l'image spéculaire}

Ces méprises causées par les mirages imaginaires n'interfèrent donc pas uniquement dans les rapports entre lofteurs, mais également dans toute vie humaine. Cette "rencontre» perpétuellement manquée avec soimême, ce «ratage» de l'être en tant que sujet indivis, débute en fait dans l'image spéculaire elle-même qui inverse latéralement la forme réelle $\mathrm{du}$ visage, reflétant ainsi une version inexacte de sa physionomie. "La re-connaissance de soi à partir de l'image du miroir s'effectue - pour des raisons optiques - à partir d'indices extérieurs et symétriquement inversés. Du même coup, c'est donc l'unité du corps elle-même qui s'ébauche comme extérieure à soi et inversée „54. Ainsi, l'image de soi que l'on va chercher dans l'Autre, non seulement nous échappe, mais s'avère originairement être déformée par l'altération énantiomorphe (inversion morphologique dans l'axe perpendiculaire à la surface du miroir) 55. Malgré cette faute d'objectivité de la part du miroir, nous nous attachons à cette image considérée comme étant la vraie version de notre visage. En effet, "nous ne sommes jamais les maîtres de ce double, dans la glace, qui représente l'Autre en nous, foncièrement décalé, et qui peut prendre une existence autonome, incontrôlable, persécutoire, ou paranoïaque »56. Ainsi, on peut dire que la fonction du miroir est essentielle car elle symbolise visuellement le rapport existentiel de l'individu à son image propre, un rapport qui est «instable, oscillant entre les pôles de l'être et du paraître, de l'objectivité et de la subjectivité, de la vérité et du fantasme, de l'intime et du collectif, etc. $\mathbf{5 7}^{\mathbf{5 7}}$

L'inversion géométrique de l'image spéculaire entérine le mirage identificatoire mis à jour par le stade du miroir, apporte une confirmation supplémentaire sur le fait que l'image de soi est fondamentalement biaisée. La géométrie semble, en effet, soutenir et approuver la psychanalyse dans son analyse de la division du psychisme humain: "l'inversion spéculaire [...] c'est une version originale, plus péremptoire que toute autre image, du leurre formateur de notre personne. Nous investissons complaisamment l'énantiomorphie du miroir en spéculant sur la commutativité de notre symétrie bilatérale sous l'effet d'une contrainte de répétition qui nous entraîne à rejouer interminablement le scénario identitaire ou le clivage inaugural» $\mathbf{5 8}$.

Les miroirs du Loft, comme d'ailleurs tous les autres, nous apprennent que le sort de l'homme c'est précisément d'être condamné à "une impropriété constitutive, c'est de n'être aucunement identique à soi, c'est de vouloir gérer cette insuperposabilité spécifiquement spéculaire 
du voir et de l'être vu, ou du pour-soi et du pour-autrui, insuperposabilité ontologique qui fait de nous des sujets clivés»59. Ainsi le miroir, en réfléchissant avant tout un porte-à-faux entre le sujet et son image, le sujet et les autres, représente "la métaphore prototypique du désir de l'autre, dans le regard duquel je retrouve ma propre demande mais sous une forme inversée "60. Ce décentrement du sujet qui est "constitué par une structure qui, elle aussi, n'a de centre que dans la méconnaissance imaginaire du moi, c'est-à-dire dans les formations idéologiques où il se reconnaît» $\mathbf{6 1}$, trouve dans le Loft une preuve éclatante. La pénitence majeure endurée par ces reclus de bonne volonté, c'est de faire publiquement et impudiquement l'expérience de l'aliénation du sujet au champ de l'Autre qui accompagne toute existence humaine.

L'analyse du dispositif du Loft réalisée dans une double perspective, à la fois historique et psychanalytique, nous a permis, on l'espère, de démontrer que ce jeu de télé-réalité constitue un objet d'étude idéal et unique pour mettre à jour certaines des fonctions du regard et du miroir. À cela s'ajoute le fait que le Loft incarne, par une mise en évidence des fantasmes d'omniperception propres à une société qui exalte les vertus de la vision totalisante, englobante et soi-disant sécurisante, le fonctionnement même de la télévision qui fait voir pour mieux se regarder elle-même. En effet, offrir la possibilité à une masse informe et sans identité d'accéder à une vision sans limites, et à quelques élus de bénéficier d'une visibilité notoire, revient à satisfaire cette obsession du voir et de l'être-vu qui animent aujourd'hui la télévision dans son ensemble. Le Loft est ainsi une sorte de caricature de la télévision elle-même qui fait ici la démonstration de sa capacité à faire voir tout... de rien, tout de suite, à tout le monde et pour rien (ou pas grand-chose). Et à Guy Debord, dont le texte de 1967 n'a pas pris une ride et qui peut être lu comme une exégèse visionnaire et brillante des jeux de télé-réalité, laissons le dernier mot. «La société qui repose sur l'industrie moderne n'est pas fortuitement ou superficiellement spectaculaire, elle est fondamentalement spectacliste. Dans le spectacle, image de l'économie régnante, le but n'est rien, le développement est tout. Le spectacle ne veut en venir à rien d'autre qu'à lui-même»62.
59 ld., p. 32.

60 ld., p. 29.

61 Louis Althusser, "Freud et Lacan" (1964), Ecrits sur la psychanalyse, Stock/IMEC, Paris, 1993, p. 45.

62 Guy Debord, La société du spectacle, Gallimard, Paris, 1992 [1967], p. 21. II est frappant de constater que, parmi les jeux préférés des lofteurs (non imposés par le "Propriétaire ", donc tout à fait spontanés), les parodies d'émissions ou de séries télévisées célèbres, ou alors des exercices typiquement télévisuels abondent: "Qui veut gagner des millions?", "Les trois drôles de dames", des faux entretiens réalisés face à une caméra, un fouet de cuisine en guise de micro, ou l'invention d'un nouveau concept d'émission, "Quatre-vingt-

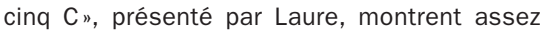
bien cette tendance à l'auto-représentation, qui dans, des émissions comme "Les enfants de la télé ", atteint des sommets d'auto-congratulation complaisante. Finalement, ce ne sont pas tant les personnes en quête de quelque gloire incertaine, que la télévision elle-même qui, à force de se regarder, souffre d'un narcissisme chronique. 